\title{
Les fonctions démystificatrices de la guerre dans les Âmes grises et Le rapport de Brodeck de Philippe Claudel
}

\section{Abstract:}

This article entitled "demystifications functions of war in gray souls and the report Brodeckto Philippe Claudel attempts to elucidate one side of the narrative strategy with what is told the theme of war. On the other hand, he tries to describe the impact of the event on the lives of the characters.

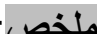

هذا المقال عنوانه الوظائف السلبية للحرب في الأرواح الرمادية وتقرير بروداك لصاحبه فيليب كلودال حيث يحتوي على شقين. أما الثق الأول فهو محاولة لبيان الإستر اتيجية التي اعتمد ت في سرد موضوع الحرب. أما الثق الثاني فهو

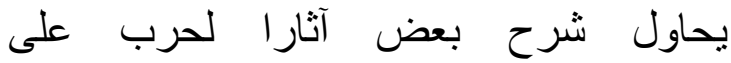

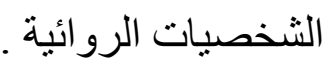

\section{ABDELLI Yacine}

Faculté des Lettres et Langues

Département de français Université Mentouri

Constantine

\section{Introduction :}

La guerre constitue irréfutablement un thème fertile pour beaucoup de poètes et d'écrivains, notamment dans l'Antiquité, où les épopées ont été conçues comme des récits. Bien qu'elles expliquent la vie de la cité, elles glorifient essentiellement les exploits politiques et guerriers de la classe dirigeante et expriment particulièrement le point de vue des puissants. Donc, l'intérêt dans la tradition épique s'inscrit dans une perspective qui offre au lecteur à travers les personnages de premier rang, vénérés comme des dieux, une image à la fois exaltante et valorisante. 
Néanmoins, ce rapport dialectique de la littérature à la guerre semble être profondément modifié, voire inversé. "L'épopée avait reçu de rudes coups dès le XVIIIème siècle، mais elle ne fut définitivement morte qu'avec l'apparition de son contraire، aux XIXème et XXème siècles' qu'on pourrait appeler l'épopée antimilitariste ou pacifiste ${ }^{(1) " . ~ C ' e s t ~ a l o r s ~ u n e ~ n o u v e l l e ~ f a c ̧ o n ~}$ d'envisager la guerre a vu le jour sous la plume entre autres de : Jean Giono(le Grand Troupeau), Céline (Voyage au bout de la nuit). Il s'agit bien évidemment dans l'examen de cette question, dans sa forme romanesque contemporaine, de déprécier la guerre, de détruire ce mythe considéré pour longtemps noble. Ainsi, pour alourdir le réquisitoire et frapper les imaginations, les auteurs peignent les guerres par les souffrances inouïes des hommes, les horreurs du front, le déchirement des couples, l'envoi à l'exil... Mais, cela n'empêche en rien de souligner, qu'à côté de ceux qui refusent cette machine qui mutile et broie, subsistent encore ceux qui acceptent de devenir les complices de la guerre.

Entre panégyristes, voyant la guerre au sens classique une source de vertus (courage, héroïsme), et ses adversaires farouches qui dénoncent son absurdité, Philippe Claudel a choisi d'être un habile détracteur de celle-ci. Pour lui, ce qui importe, ce n'est pas l'événement historique en soi, mais, ce sont les raisons et les incidences de celui-ci sur la formation des personnages. Car, en évoquant soit l'affaire Dreyfus, soit les deux évènements majeurs du $\mathrm{XX}^{\mathrm{ème}}$ siècle, à savoir la $1^{\text {ère }}$ et la $2^{\text {ème }}$ guerre mondiale, il n'a en aucun cas la prétention de rivaliser les historiens dans leur domaine.

Pour lui, la matière historique est saisie pour d'autres fins que la pure connaissance. Encore, faut-il ajouter que le terrain de prédilection pour l'auteur des Âmes grises n'est pas le front, là où les instincts sont poussés à leur paroxysme et s'élèvent à une vertu surhumaine. Par ailleurs, il s'abstient de décrire soit la vie des soldats dans les tranchées, soit le déroulement des batailles comme l'ont fait ses prédécesseurs (dans ce cas, la bonne illustration en est Le Feu d'Henri Barbusse). Ce sont là, nous semble-t-il des thèmes usés. Cependant, le vrai combat est à mener, sur le terrain des valeurs, contre les désirs qui hantent les âmes et les fausses idées qui nourrissent les pensées.

Bien qu'elle soit mise en arrière-plan et confinée à un espace étroit, la guerre comme thème unissant les deux textes, demeure en revanche l'élément déterminant qui organise sa configuration et jalonne les parcours narratifs des personnages. A cet effet, nous tenterons dans cet article d'élucider les manifestations de la guerre, et dire, dans quelle perspective s'inscrivent-elles ? A cela, il convient de répondre à cette interrogation : quels rôles assignonsnous à la guerre dans les deux romans de P. Claudel ? En d'autres termes, quels sont les éléments mis en œuvre pour condamner cette machine destructrice?

La guerre, un élément révélateur, et découverte de soi 
Les sentiments de haine, de mépris, d'avilissement..., naissent naturellement avec l'homme, se dessèchent en temps de paix, grandissent et s'épanouissent, cependant aux moments exécrables de la guerre. De ce fait, les égarements ou les folles passions qui apparaissent, aussi bien dans Les Ames grises que dans Le rapport de Brodeck, comme des attitudes incompréhensibles des personnages, s'alimentent en partie par le contexte de guerre qui les entoure. C'est dans ces circonstances que le mal de l'homme trouve sa matière, s'aggrave puis devient plus lourd à supporter. A cet égard, il semble bien que la guerre ne crée ni les comportements des uns, ni les raisonnements des autres, mais, seulement elle leur permet de se manifester. " Dans l'ensemble ‘ la guerre fournit (...) des aliments au snobisme et aux passions; elle ne modifie que leurs manifestations (2)"

C'est également ce qu'éprouve cruellement le narrateur dans Les Ames grises quand il dévoile la part obscure, la véritable profondeur de l'âme humaine dans sa réaction sous le péril. Celle-ci nous délivre ses états latents et morbides:

" On tue beaucoup dans une journée, sans même se rendre compte vraiment, en pensée et en mots. Au regard de tous ces crimes abstraits, les assassinats véritables sont bien peu nombreux, si l'on y réfléchit. Il n'y a vraiment, que dans les guerres que l'équilibre se fait entre nos désirs avariés et le réel absolu ${ }^{(3) "}$ ".

Une fois de plus, le sens révélateur apparaît explicitement dans Le rapport de Brodeck, par la voix d'un important personnage de l'univers romanesque, le Maire Orschuir qui s'exprime sous une forme versifiée : "La guerre ravage et révèle ${ }^{\prime(4)}$ Sans doute, la guerre autant qu'elle laisse des traumatismes derrière elle, autant qu'elle rend visible les choses qui se sont endormies : LLa guerre permet en effet d'accéder à la face cachée des choses ${ }^{(5) " . ~}$

Comme elle ravive les haines, exacerbe les souffrances, la réalité guerrière ne doit pas bien entendu masquer la générosité des individus, qui, à l'opposé de ce tableau brossé en noir peut de même réveiller chez l'homme les sentiments les plus nobles : amour, solidarité...A cet effet, le narrateur dans Les Ames grises évoque le personnage de madame De Flers venue du beau monde, par souci d'abnégation, elle veut collaborer bénévolement aux soins des blessés et veiller au chevet des mourants. " Certains ont dit: " Elle restera pas trois jours ‘ la vue $d u$ sang et de la merde la fera tomber dans les pommes "(6)

"Elle est restée. Malgré le sang et la merde, faisant oublier sa particule et sa fortune par une bonté sans limites et des gestes simples(...), passait ses nuits au chevet des mourants et des ressuscités. La guerre massacre, souille, salit, éventre, sépare, hache, tue, mais parfois, elle remet aussi certaines pendules à la bonne heure ${ }^{(7) "}$.Au demeurant, faut-il rappeler que l'homme est pétri de bien et de mal, et que sous les menaces de la guerre, il délivre l'un comme l'autre Dans cette même optique, qui consiste à insister sur l'aspect " révélateur " de la guerre, est-il possible d'ignorer les clivages des opinions, les divisions 
d'intérêts, les querelles intestines du même groupe social ou de la même nation en temps de paix ?Il serait aussi vain que stérile d'approuver cette vision platonique selon laquelle les membres d'une communauté ou d'une nation forment un tout, parfaitement cohérent et ne portent ni germes de dissension, ni présentent des signes d'une injustice sociale. Cependant, si ces indices sont peu perceptibles ou difficilement reconnaissables en temps de paix, ils deviennent bien évidemment plus criants, voire plus scandaleux en temps de guerre. " Le scandale de la guerre tient sans doute pour une part non négligeable ‘̀ son caractère inhumain. Elle est intrinsèquement mauvaise. Mais elle est surtout l'expression grossie d'un ordre humain odieux, de l'injustice sociale fondamentale qu'elle révèle (au sens photographique du mot)(8)".

Si nous considérons La semaine sainte de Louis Aragon, l'exemple parfait qui appartient au sens générique aux romans d'apprentissage historique, et ceux qui rapportent l'expérience militaire des personnages, comme des romans de témoignage, ceux en revanche, des Âmes grises et Le rapport de Brodeck permettent la découverte de soi, ainsi que la découverte des contraintes de la société. Ce qui assigne par ailleurs à la guerre un rôle de loupe qui consiste à mettre la lumière sur l'instinct guerrier et révèle le délire des foules. A cet effet, l'examen du parcours narratif d'Ulli Rate, dans Le rapport de Brodeck, illustre clairement l'impact de la circonstance sur le retournement total d'attitude de celui-ci. Sachant que, ce dernier ami de Brodeck ,pourtant connu par son caractère conciliant durant son passage à l'université, mais, devient l'une des pièces d'une machine qui sème la mort, un tortionnaire qui pratique les supplices dans les camps d'extermination: " L'étudiant Ulli Rate était devenu un des gardes du camp, c'est-à-dire une des pièces parfaitement huilées et obéissantes (...) d'une machine dans laquelle on nous enfournait ${ }^{(9) "}$

"(...) que cet homme (Ulli Rate) grand et gras، heureux d'être un bourreau $(\ldots)^{(10) "}$

Pourquoi Ulli s'est-il brusquement métamorphosé, d'un jeune jovial aimant la vie à un bourreau ? Quel mobile l'a poussé à devenir un serviteur d'un système qui broie les hommes. Pour répondre à cette question il convient de rappeler que la guerre représente simplement une circonstance dans laquelle se révèle le bas fond des hommes, comme l'atteste Maurice Rieuneau" La guerre ne dégrade pas les hommes, elle les révèle tels qu'ils sont ${ }^{(11) " .}$

\section{La guerre, dessine une sorte de manichéisme social}

Le narrateur dans Les Âmes grises nous dépeint l'univers de cette petite et paisible ville qui vit en retrait du monde. Seulement, il semble bien que la guerre a eu ses effets sur ces habitants. Car, elle a bouleversé de fond en comble leur vie, et a creusé entre eux, non pas un fossé mais un abîme. " Il y eut alors comme deux villes ' la nôtre, et la leur. Deux villes au même endroit, mais qui se tournaient le dos, avaient leurs promenades, leurs heures. Deux 
mondes. Il y eut même des insultes, des coups de gueule, des coups de poing ${ }^{(12) "}$.

$\mathrm{Au}$ fond, le narrateur nous dessine une sorte de manichéisme social, selon lequel la ville est divisée en deux mondes. Le premier, représente celui des non-mobilisés et leurs familles, tandis que le deuxième, c'est celui des mobilisés. Ainsi, toute la société se retrouve à travers le spectre de la guerre scindée en deux, celle du front, et celle de l'arrière avec deux catégories de personnages antagonistes, ceux qui jouissent, c'est-à-dire vivant à l'abri du danger et ceux qui souffrent. Parmi les images qui s'offrent à l'esprit des soldats, ce sont les délices de l'arrière : le chaud des lits, le parfum des femmes et le sommeil, contre toutes les cruautés du front, là où se mêlent la peur et l'aversion:" Huit cent hommes qui aux yeux de certains n'en furent jamais et qui, chaque matin, sortiraient d'un lit chaud, de bras endormis, et non d'une tranchée boueuse, pour aller pousser des wagon nets( il s'agit des ouvriers) plutôt que de cadavres. La bonne aubaine! Le souffle des obus, la peur, les copains qui geignent et meurent à vingt mètres accrochés dans les barbelés, les rats rongeant les morts, au loin tout ça! A la place, la vie, la vraie, tout simplement. La vie chaque matin étreinte non pas comme un rêve par-delà les fumées mais comme une certitude chaude qui sent le sommeil et le parfum des femmes "Veinards! Planqués!"(13)"

Notons au passage, que rien ne serait plus faux d'ignorer la pensée du narrateur et d'insérer les deux extraits cités ci-dessus uniquement dans une perspective descriptive qui consiste à établir un rapport de comparaison entre le front et l'arrière. Car, il nous semble que l'opinion du narrateur parait toute proche des gens de l'arrière plutôt que ceux du front avec lesquels, il marque une certaine distance, notamment quand il fait usage du pronom possessif " nôtre » et dit: "Il y eut alors comme deux villes, la nôtre, et la leur (14)"

Mais, faut-il ajouter, que cette dualisation de la société, secrétée par la guerre et dans laquelle coexistent deux attitudes irréductibles, d'un côté les acteurs du front, de l'autre, les civils considérés comme les complices de la guerre, ne s'estompe ni s'efface à l'aube d'un cessez-le-feu. Paradoxalement, il serait crédule de songer que, ses rapports tendus, régis par une haine mutuelle durant les conflits, prennent fin une fois les belligérants suspendent leurs hostilités. A vrai dire, le tragique de la guerre n'est jamais effacé, les rancunes sont durables, elles l'emportent souvent sur les motifs de l'unisson et la cohésion sociale "Des fils revinrent et des maris. Certains intacts, d'autres bien allumés .Beaucoup ne réapparurent jamais (...) les familles qui avaient les leurs à l'Usine avaient traversé la guerre sans trop de soucis ni de privation. Les autres par contre sortaient de quatre années terribles. Le fossé se creusa plus encore, surtout lorsqu'un mort ou deux achevaient d'y pourrir. Certains ne se parlèrent plus. D'autres en vinrent à se haïr ${ }^{(15) " . ~}$ 
Toutefois, si les obus cessent d'être lancés, ce n'est pas dans un proche avenir que les ferments de la haine disparaissent. Même après une déclaration de fin de guerre, celle-ci n'arrêtera certainement pas les comportements belliqueux des uns et des autres. Ses attitudes continueront d'être éveillées et trouveront leurs éléments féconds dans les graines de violence que la guerre a semées .Et tant que les plaies persistent et restent ni pansées, ni définitivement guéries, l'édifice social demeure continûment vacillant surtout quand ce dernier est mis aux rudes épreuves, même en temps de paix.

La guerre, un prétexte pour exprimer le naufrage des valeurs

A la différence d'Henri Barbusse lequel préfère décrire la réalité des combats et ses scènes apocalyptiques : corps déchiquetés, organes mutilés, et cris de détresse poussés, la réflexion claudélienne sur ce thème prend une autre proportion, dépassant amplement les contours du front, pour s'inscrire dans un autre espace bien vital, mais, longtemps négligé ou jeter aux oubliettes. Celui de la réalité des gens de peu, ceux qui ne participent pas à la guerre, mais, qui subissent sans comprendre le contrecoup de ce désastre. Donc, cette approche prend sa vraie signification au contact de ses humbles âmes qui sont réduites à la misère noire, à l'humiliation et au malheur.

Bien que l'arrière ne soit le théâtre des affrontements, il est bel et bien envahi par l'ombre de la guerre. Cette dernière apparait seulement comme toile de fond dans les deux textes, néanmoins, elle fournit un bon prétexte, ou une occasion combien propice pour, porter un regard sévère sur les mœurs les plus étranges, en réprouvant les hiérarchies communément admises et en exposant au grand jour un système d'idées, à travers lequel l'auteur fustige d'une manière à la fois tacite et explicite, l'ordre guerrier, ainsi que ses répercussions dramatiques, sociales, morales et humaines.

Face à cette institution destructrice multiforme qui n'est avant tout qu'une pure création humaine, nombreux sont les cris de colère, de refus qui s'élèvent et retentissent dans Le rapport de Brodeck et Les Âmes grises, et qui témoignent d'un total désaveu, et de l'extrême dérision pour ce drame, ces cris traduisent remarquablement une conscience démystificatrice dans ses textes.

En effet, pour condamner, dans toute la force du terme, l'ordre guerrier, Brodeck le protagoniste s'interrompt pour laisser entendre le discours du prêtre Peiper, qui résume sans détour cette malédiction de guerre qui s'abat sur ces pauvres gens: "Sept ans de malheur! Sept ans de malheur! Sept ans de malheur!"(16).

Il s'agit au passage, comme il convient de le souligner d'une allusion faite au drame de la $2^{\text {ème }}$ guerre mondiale (1939-1945) qui a duré sept ans. Ainsi, pour donner un poids sémantique accru à ses propos, et insister sur le sens de "malheur", l'instance narrative a fait usage d'une figure de construction qui est la répétition lexicale, dont l'une de ses variantes comme 
dans cet extrait, est la réduplication ${ }^{(17)}$." Celle-ci consiste à redoubler dans le même membre de phrase quelques mots d'un intérêt marqué. "

A vrai dire ce qui nous semble important, c'est sans doute, ce choix porté sur cette autorité morale, au nom de Peiper, pour dénoncer clairement cette malédiction de la guerre. En fait, si d'autres voix du paysage narratif sont considérées comme complices par leur silence et restent muettes sur ce sujet. Celle de l'église en revanche, s'élève contre vents et marées pour marteler nettement son mot, car cette voix de sagesse, jouit d'une crédibilité assez conséquente pour persuader.

Au-delà des cris qui s'élèvent haut et fort dans l'écriture claudélienne condamnant sans équivoque la guerre qui rappelons-le, n'est envisagée que comme un bruit de fond, ou comme une occasion exceptionnelle, ce sont plutôt les incidences de celle-ci sur la vie des personnages qui priment. Initialement, la guerre fournit le contexte, mais ensuite elle cède la place à ses retentissements tels que : l'écroulement de la civilisation, l'effondrement des conventions sociale et morale et le dépaysement que subissent les malheureux personnages. Tous ces effets nous enseignent sur le non-sens, la barbarie et l'absurdité de la guerre. "La guerre est tout ce qu'on ne comprend pas "(18).

D'ailleurs, ce qui relève de l'incompréhension et du paradoxe, c'est cet esprit empêtré dans la contradiction. Dans un premier temps, il peine pour construire et pour progresser, et dans un deuxième temps, il détruit, saccage ses biens et son patrimoine. Plus significatif encore, sont les propos du prêtre Peiper qui s'exprime avec acuité sur cette irrationalité dans une longue discussion avec Brodeck: "La guerre ...peut-être les peuples ont-ils besoin de ces cauchemars. Ils saccagent ce qu'ils ont mis des siècles à construire. On détruit ce qu'hier on

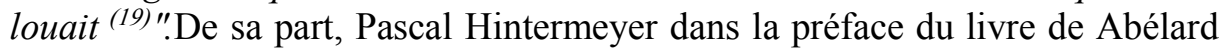
Mashimango intitulé La dimension sacrificielle de la guerre pose cette problématique et s'interroge "comment comprendre que des sociétés qui attachent en temps de paix tant d'importance à l'accumulation des richesses et à la préservation de la vie humaine sacrifient périodiquement ces valeurs en ce passionnant pour ce qui les contredit ${ }^{(20)}$ ?"

Face à un tel paradoxe, qui exprime le non-sens de la guerre, il serait inconcevable qu'un pareil effort conjugué à l'intelligence humaine durant des lustres tourne à une sorte de pensée autodestructive. Car, il se trouve que dans l'expérience humaine, aucun des belligérants ne s'en sorte indemne d'une telle situation. De ce fait, l'esprit est sollicité à s'exprimer sur deux idées antithétiques, et faire son choix. Soit sur la première thèse qui consiste à apporter à l'édifice du savoir, et de la civilisation sa pierre, afin de garder à l'abri les acquisitions des sociétés humaines .Soit sur la deuxième, considérée comme l'antithèse de la précédente, et dont les partisans ne refusent pas seulement de collaborer dans l'œuvre collective, mais deviennent l'ennemi juré de tout progrès, ils poussent à la destruction méthodique du capital de 
civilisation acquis après tant d'années, ou de siècles de labeur. "La guerre n'est pas racontée comme une expérience limitée et achevée, formant un tout isolant (...).elle devient plutôt l'ouverture ou le tremplin vers une philosophie totale à la vie, l'expérience cruciale qui met en question l'idée du bonheur, de l'action, de l'histoire, de la société(21).

Cette mise en valeur par contraste, nous semble s'insérer dans une méditation morale, elle laisse entendre en sourdine un appel à la réflexion, à la sagesse .Celle-ci, est plus que jamais invitée à faire la part de ce déchaînement des passions, de l'instinct guerrier sur la vie de l'esprit. Sachant bien que, l'appel à cette conscience vient d'un personnage privilégié, le prêtre Peiper ,qui mérite bien évidemment un intérêt particulier. Ainsi, pour mettre à nu cette macabre machine qui "gaspille tout, le sang, la vie, le courage ${ }^{(22) " c o m m e ~ d i s a i t ~}$ Duhamel, l'accent est mis sur ses dévastations, sur ses ruines, sur ses dégradations qui ramènent l'humanité à une ère primitive. En somme, pour restituer à la guerre son sens funeste, c'est sur son caractère absurde que se focalise toute l'attention. Alors, dans une première lecture la guerre est dénoncée, par cette image de référence négative qui s'offre à la pensée. Mais, dans une deuxième, cette condamnation par l'absurde s'entend comme étant une aspiration pacifique, ou une proposition de bons offices pour rétablir la paix.

Si dans l'écriture claudélienne, la guerre est l'expression du non-sens, dans laquelle les personnages demeurent une proie facile à la barbarie, elle constitue également le champ d'une manifestation accrue de souffrances, de privations et de discriminations sociales. A cet égard, le narrateur dans Les Âmes grises, nous brosse un tableau hurlant qui raconte l'une des facettes de la vie de l'infortuné médecin du village, Hippolyte Lucy, un personnage lucide et humain. Ce dernier, autre du dévouement qu'il offre à ses patients, s'abstient de percevoir volontairement une contrepartie financière"(...) car rarement il se faisait payer la visite quand il allait chez les gens de peu ${ }^{(23) " .}$

En plus, il joint le geste à la parole en leur disant "vous me paierez plus tard !", faisant ainsi jaillir une bonté sans limites, dans une période de disgrâce. Mais, à l'inverse de cette sorte d'admiration à la belle figure de Lucy "Un bon docteur $^{(24)}$ ", le tragique de la guerre gagne de plus en plus le terrain, il s'empare davantage à de vastes secteurs de la société, même longtemps après un simulacre de cessez-le feu, les peines et les traumatismes subsistent, faisant presque de l'ensemble des personnages forcément des nécessiteux, des gens misérables, sinon comment expliquer cette triste fin du docteur Lucy ?"(...) c'est la misère qui l'a tué (...) (25)".

Donc la guerre a brisé un homme intelligent, philanthrope qui a mis tous ses efforts dans une œuvre de médecin. Avec cette illustration et bien d'autres, Claudel rejoint, l'idée d'Eugène Dabit dans Petit Louis, que seuls intéressent, les sujets des pauvres réalités. "Je (Dabit) préfère choisir ces images qui sans 
être sanglantes, n'en laissèrent pas moins dans mon esprit des traces profondes ${ }^{(26) "}$.

Au demeurant, évoquer ces images inspirent la pitié, cela relève du caractère pathétique de l'œuvre, qui fait naître une certaine sympathie pour ces personnages accablés de malheur .Mais conjointement, elle interroge la conscience pour y émettre ses jugements sur les valeurs sociales et morales bouleversées de fond en comble. "La guerre, c'est une grande main qui balaie le monde. C'est le lieu où triomphe le médiocre, le criminel reçoit l'auréole du saint, on se prosterne devant lui, on l'acclame, on l'adule. (27)". Presque, de bout en bout dans le rapport de Brodeck, quand le prêtre Peiper s'exprime sur le sujet de la guerre, c'est pour la dénoncer et montrer combien cette machine intrinsèquement destructrice crée des situations considérablement imprévues et imprévisibles. Pour lui, elle est l'origine de tout changement brutal que subissent les personnages, mais provoque aussi le renversement total des normes sociétales. "La guerre, qui brise les barrières morales et les interdits sociaux, produit un dépaysement, une mutation brusque des conditions de vie, et par là une occasion imprévue pour les appétits instinctifs ${ }^{(28) " .}$

Nous pouvons dire que, quelle que soit la puissance d'une nation, sa prédominance reste passagère ou temporelle, cependant les conséquences de l'événement demeurent, et pèseront lourdement sur la postérité. Car ce chamboulement des valeurs au moment des guerres laisse derrière de violentes et profondes divisions, d'intérêts, de convictions et de sentiments .Et la seule chance de salut comme disait M.Rieuneau c'est "Dans un retour résolu aux valeurs bafouées de la civilisation humaniste (...). ${ }^{(29) " C a r}$ selon lui encore :"La guerre est née d'un oubli de ces valeurs' qui équivaut à un reniement de la civilisation même $e^{(30) "}$. Donc ce qui apparaît important dans l'écriture claudélienne, ce n'est pas assurément les faits de guerre, à savoir les exploits des armées, les prouesses des soldats, comme le courage et l'héroïsme, mais, ce sont plutôt ses effets, ses conséquences qui ramènent la condition humaine à un âge de barbarie. En d'autres termes, l'événement compte moins pour Claudel que les résultats qu'il entraine.

Enfin, nous pensons que, ces manifestations paraissent difficilement décelables dans d'autres situations moins tumultueuses. Seule la guerre, ou une circonstance pareille permet de dévoiler la part obscure des choses. A cet égard ,les quelques fonctions de la guerre que nous venons d'examiner dans ces deux textes s'inscrivent dans une perspective dont les traits les plus saillants sont, la révélation de l'image latente des individus, la conception dualiste de la société(Face à la guerre, les citoyens sont scindés en deux catégories, celle des bons, et celle des mauvais), et enfin, le naufrage des valeurs (la guerre est synonyme d'absurdité, 
du non-sens...).Ici, Claudel met le doigt sur le mal, le situant ainsi au cœur de l'homme. Dans ce cas, s'il est au fond de l'homme, pouvons-nous changé l'homme

\section{Bibliographie}

\section{1/ Euvres analysées}

Philippe Claudel -Les Âmes grises, Paris stock, 2003.

-Le rapport de Brodeck, Paris, stock.2007.

\section{2/ Ouvrages théoriques}

1/Abélard Mashimang Abou-Bakr, la dimension sacrificielle de la guerre, essai sur la martyrologie politique, Paris, l'Harmattan, 2012.

2/ Guénon René, la crise du monde moderne, Paris, Gallimard, 1973

3/ Rieuneau Maurice, guerre et révolution dans le roman français de 1919 à 1939, Genève, Slatkine repeints, 2000.

4/Sabot Philippe, littérature et guerre, Sartre, Malraux, Simon, Paris, PUf, 2010.

\section{3/ Dictionnaires}

1/Dictionnaire de langue française, Le Petit Robert, éd. Le Robert, 2002.

2/Alain Viala et al, le dictionnaire du littéraire, Paris, PUf, 2012.

Introduction . .1

La guerre, élément révélateur, et découverte de soi...........................2

La guerre, dessine une sorte de manichéisme social.........................5

La guerre, un prétexte pour exprimer le naufrage des valeurs..................... 6

Conclusion............................................................... 11

Notes :

1-Maurice Rieuneau, Guerre et révolution dans le roman français de 1919 à 1939, Genève, Slatkine Repeints,2000,p.168 .

2-Ibid., p.118.

3-Philippe Claudel, Les Âmes grises, Paris, Stock, 2003, p.147. 4-P. Claudel, Le rapport de Brodeck, Paris, Stock, 2007, p.335.

5-Philippe Sabot, Littérature et guerres, Sartre, Malraux, Simon, Paris, Puf, 2010, p.7.

6-P. Claudel, Les Âmes grises, op.cit., p.203.

7-Ibid., pp.203-204.

8-Maurice Rieuneau, op.cit., p.201.

9-P. Claudel, Le rapport de Brodeck, op .cit., p.235.

10-Ibidem.

11-Maurice Rieuneau, op.cit., p.201

12-Philippe Claudel, Les Âmes grises, op.cit., p.137.

13-Ibid., p.48 
14-Ibid., p.137

15-Ibid., p.225.

16-Philippe Claudel, Le rapport de Brodeck, op.cit., p.167.

17-Catherine Fromilhagne, Les Figures de style, Paris, A. Colin, 2013(2éd), p.27.

18-Michel Raimond, Le Roman, Paris, A. Colin, 2011, p.87.

19-Philippe Claudel, Le rapport de Brodeck, op.cit., p.164

20-AbelardMashimango, La dimension sacrificielle de la guerre, Paris,

L'harmattan, 2012, préface. P.13.

21-Maurice Rieuneau, op.cit., p.341

22-Le Petit Robert, Paris, Edition Robert, 2002, p .1164.

23-P. Claudel, Les Ames grises, op.cit., p.96.

24-Ibidem.

25-Ibidem.

26-Maurice Rieuneau, op.cit., p.271.

27-P. Claudel .Le rapport de Brodeck, op.cit., pp164.165.

28-M. Rieuneau op.cit., p.110.

29-Ibid., p.260.

30-Ibidem. 